\title{
Dietary practices and beliefs of patients with inactive ulcerative colitis: A prospective study from the United Kingdom
}

\author{
B. Crooks ${ }^{1,3}$, J.K. Limdi ${ }^{2,4}$ and J.T. McLaughlin ${ }^{1,3,4}$ \\ ${ }^{1}$ The University of Manchester, Division of Diabetes, Endocrinology and Gastroenterology, Manchester, UK, \\ ${ }^{2}$ The Pennine Acute Hospitals NHS Trust, Section of IBD - Division of Gastroenterology, Jericho Road, Bury, \\ Manchester, UK, \\ ${ }^{3}$ Salford Royal NHS Foundation Trust, Department of Gastroenterology, Salford, UK and \\ ${ }^{4}$ Manchester Academic Health Science Centre, Manchester, UK
}

An epidemiological association implicating diet in inflammatory bowel disease (IBD) risk or protection is widely accepted and patients often make links to diet. Recent studies have explored dietary habits in the general IBD population but few studies have specifically addressed this in active or inactive disease ${ }^{(1)}$. We aim to describe the dietary practices of patients with inactive ulcerative colitis (UC) and the information resources which guide these beliefs.

We developed a questionnaire assessing demographics, dietary beliefs and habits in patients with inactive UC (defined as a PRO2 score $\leq 1$ (rectal bleeding sub score $=0))^{(2)}$. This is being prospectively administered to 200 consecutive patients with inactive UC. We report an interim analysis of our findings.

Data from 108 patients; mean age 55 years, 61\% male and 91\% Caucasian, are available so far. Mean disease duration was 13 years. Disease extent was 16\% E1, 44\% E2 and 30\% E3. Mean CRP was 3.7. 61\% of patients reported no relapse of their UC in the last year. $29 \%$ considered diet to be an initiating factor for their UC and this was based on their own experience ( $97 \%)$, information found on the internet $(29 \%)$ and the advice of a gastroenterologist $(26 \%)$.

$40 \%$ of patients felt that diet had triggered a relapse of their disease, the most commonly reported triggers being spicy foods (51\%), alcohol $(35 \%)$, fatty foods $(33 \%)$ and coffee $(26 \%) .55 \%$ of patients avoided certain foods, at least sometimes, to prevent relapse. Spicy $(73 \%)$ and fatty $(63 \%)$ foods were most frequently avoided followed by alcohol $(50 \%)$, coffee $(45 \%)$, carbonated drinks $(42 \%)$, milk products $(42 \%)$ and red meat $(35 \%)$. Female patients were significantly more likely to restrict their diet in an attempt to prevent relapse of their UC $(p=0.04)$.

A fifth of patients consumed specific foods or nutritional supplements in an attempt to prevent UC relapse, most commonly turmeric and probiotics. Less than half of patients reported being able to find specific dietary advice for UC and this was most commonly sourced via the internet through organisations such as Crohn's and Colitis UK.

A significant proportion of patients with inactive UC have strong beliefs pertaining to the role of diet and specific dietary triggers in the initiation of disease and subsequent risk of relapse. A high prevalence of dietary restriction may put these patients at risk of nutritional deficiency and impact upon quality of life. More data and mechanistic explanations are needed to understand the complicated relationship between dietary components and gut inflammation. A larger dataset will be presented at conference proceedings.

Support provided by Takeda

1. Limdi JK, Aggarwal D, McLaughlin JT (2016) Inflamm Bowel Dis 22, 164-170.

2. Jairath V et al. (2015) Aliment Pharmacol Ther 42, 1200-1210. 